\title{
Prevalence and Correlates of Overweight and Obesity among Preschool-Aged Children in San Luis Obispo County, 2006-2014
}

\author{
Kristine Z. Jankovitz ${ }^{1}$, Alison K. Ventura ${ }^{1}$, Trevor M. Curry ${ }^{1}$, Victoria B. Howarth ${ }^{1}$, Caitlin C. \\ Moran ${ }^{1}$, Robin M. Mertens ${ }^{1}$, Leland A. Bailey ${ }^{2}$, Kristen Thompson ${ }^{1}$, and Karen McGaughey ${ }^{2}$ \\ ${ }^{1}$ Department of Kinesiology and Public Health, California Polytechnic State University, San \\ Luis Obispo \\ ${ }^{2}$ Department of Statistics, California Polytechnic State University, San Luis Obispo
}

\begin{abstract}
Background and Purpose: National prevalence of overweight and obesity (OW/OB) among children remains high; surveillance of $\mathrm{OW} / \mathrm{OB}$ at state- and local-levels is needed. This study determines the prevalence and sociodemographic predictors of OW/OB among preschool-age children in San Luis Obispo (SLO) County. Methods: Cross-sectional convenience samples of Head Start, California State, and private preschools were surveyed in $2006(n=482), 2009 / 10(n=559)$, and $2014(n=442)$. At all waves, preschool children aged 3-5 years were measured for height and weight using standardized stadiometer and digital scale protocols. In 2014, parents completed a demographics questionnaire. Results: Children assessed in 2009/10 and 2014 were more likely to be OW/OB than those assessed in 2006 ( $\mathrm{p}=0.016$ ). Unadjusted, bivariate odds ratios illustrated increased risk for OW/OB was associated with Hispanic ethnicity, residing in a lower income household, attending preschool in southern SLO County, and participation in a Head Start preschool. In the adjusted, multivariable model, increased risk for OW/OB was associated with attending preschool in SLO City and participation in a California State or Head Start preschool. Conclusion: OW/OB trends in SLO County are similar to national trends. Programs to continue to monitor and reduce socioeconomic disparity in OW/OB prevalence among young children are needed.
\end{abstract}

(C) 2018 Californian Journal of Health Promotion. All rights reserved.

Keywords: overweight, obesity, prevalence, children, sociodemographic disparity

\section{Introduction}

The prevalence of overweight and obesity (OW/OB) in the United States remains high, with most recent estimates illustrating that $35 \%$ of children and adolescents are considered OW/OB (Skinner, Ravanbakht, Skelton, Perrin, \& Armstrong, 2018). Given the prevalence of OW/OB increases with age and weight status tracks between early childhood and adulthood (Freedman et al., 2005), screening and identification of predictors of heightened risk early in life are an important foundation for prevention efforts.

Significant sociodemographic disparities exist for OW/OB risk among young children (Skinner et al., 2018; Wang \& Beydoun, 2007). For example, national data illustrates significantly higher prevalence of $\mathrm{OW} / \mathrm{OB}$ for children who

are Hispanic and non-Hispanic black compared to children who are non-Hispanic white (National Center for Health Statistics, 2016; Skinner et al., 2018). Additionally, by 2 years of age, children in the lowest socioeconomic status (SES) classification have significantly greater odds of being OW/OB than children in the highest SES quintile, but this association is most pronounced among Hispanic children with a lower SES (Jones-Smith, Dieckmann, Gottlieb, Chow, \& Fernald, 2014). These findings emphasize the need to continue surveillance of $\mathrm{OW} / \mathrm{OB}$ in places where there are high densities of racial/ethnic minorities and large disparities in SES. 
In San Luis Obispo (SLO) County, approximately one third of the population is Hispanic and $15 \%$ of the population are considered to be persons in poverty (U.S. Census Bureau, 2017). We have been tracking the prevalence of OW/OB among preschool-age (3-5-year-old) children since 2006 (Jankovitz, McGaughey, Torn, Ravalin, \& McDermott, 2012). Between 2006 and 2009/2010, the prevalence of OW/OB among preschoolers in SLO County rose from $26.4 \%$ to $34.8 \%$. Additionally, SES and racial/ethnic disparity in prevalence of OW/OB emerged in the 2009/2010 survey in that children who attended Head Start preschool were 1.7 times more likely to be $\mathrm{OW} / \mathrm{OB}$ than children enrolled in private preschools and Hispanic children were 2.7 times more likely to be OW/OB than their white peers. The purpose of the present study was to estimate the prevalence of OW/OB in 2014 and combine these data with previously collected data to describe changes in the prevalence of $\mathrm{OW} / \mathrm{OB}$ since 2006. We also aimed to explore associations between weight status and ethnicity, household income, and location and type of preschool in 2014.

\section{Methods}

\section{Participants and Recruitment}

Data were collected in three waves: April-May 2006 ( $\mathrm{n}=482$ ), April-May 2009 and 2010 ( $\mathrm{n}=$ 559), and April-June $2014(\mathrm{n}=442)$. Data from the 2006 and 2009/10 assessments have been published previously (Jankovitz et al., 2012). During each wave, the SLO County Public Health Department invited all licensed public, private, California State, and Head Start preschools in SLO County to participate through a Public Health Department maintained listserv. Preschool site directors decided whether or not their school site would participate in the study and facilitated study recruitment via distribution of a recruitment letter from the Public Health Department, inclusion of study information in school newsletters, and verbal requests to participate from staff. In 2006 and 2009/10, 37 preschools participated in the study, including 7 Head Start and 12 California State Preschools; this sample comprised $57 \%$ of the 65 licensed preschool centers in the county during those waves of data collection. In 2014, 28 preschools participated, including 6 Head Start and 7 California State Preschools, which comprised $33 \%$ of the 86 licensed preschool centers in the county during that wave.

Only 3-5-year-old children whose parents or guardians agreed to their study participation and provided informed consent participated in this study. Informed consent forms and all study materials were written at a sixth-grade reading level and were available in English and Spanish. All study procedures and documents were approved by the Institutional Review Board at California Polytechnic State University, San Luis Obispo.

\section{Measures}

Trained research assistants collected all measures using standardized protocols.

Anthropometrics. Protocols for assessing children's height and weight were modified from the National Health and Nutrition Examination Survey (NHANES) (Centers for Disease Control and Prevention, 2007). Standing height was measured in duplicate to the nearest $0.1 \mathrm{~cm}$ by a portable stadiometer (Seca 214, Hamburg, Germany). Weight was measured in triplicate to the nearest $0.1 \mathrm{~kg}$ using a portable digital scale (MED-WEIGH MS-3200, Linwood, NJ). Children were assessed without shoes and in light clothing.

Children's height and weight measurements were used to calculate body mass index $\left(B M I=\mathrm{kg} / \mathrm{m}^{2}\right)$. BMIs were then converted into sex-specific percentiles using the 2000 Centers for Disease Control and Prevention (CDC) BMIfor-age growth charts for children 2-20 years. Underweight was classified as $<5^{\text {th }}$ percentile. Children in the $5^{\text {th }}-84.9^{\text {th }}$ percentiles were classified as normal weight, $\geq 85^{\text {th }}$ percentile were classified as overweight, and $\geq 95^{\text {th }}$ percentile were classified as obese.

Demographic Questionnaire. Parents completed a written questionnaire that provided additional information about their child's ethnicity, language spoken at home, and annual 
household income. Preschool staff gave questionnaire packets to parents; parents then opted to complete the survey at the preschool or to take the survey home and return it to their respective preschool when completed. Research assistants later retrieved completed surveys from preschool staff.

\section{Statistical Analyses}

All analyses were carried out using JMP Pro Version 11.1.1 and SAS Version 9.4. Seven children were classified as underweight and were, therefore, excluded from the statistical analysis. Rates of missing data were low $(<.01 \%)$; thus, only complete cases were included. Family income categories were created based on quartiles of the data. Descriptive statistics were used to summarize the prevalence of OW/OB by year, ethnicity, household income, and location and type of preschool. Logistic regression analysis was used to compare risk for OW/OB by each year of the study.

In our previous paper, we examined whether risk for OW/OB was associated with child sex and sociodemographic characteristics for the children assessed in 2006 and 2009/10 (Jankovitz et al., 2012). In the present study, we conducted logistic regression analyses that only included the children assessed in 2014 to similarly compare risk for OW/OB by child sex and sociodemographic characteristics (ethnicity, region, type of preschool, and annual family income) in 2014. For the sociodemographic characteristics (ethnicity, region, type of preschool, and annual family income), we initially conducted bivariate analyses to examine the association between each characteristic and OW/OB individually. We then included all sociodemographic characteristics into a single multivariable model describing risk for OW/OB by ethnicity, region, type of preschool, and annual family income.

\section{Results}

\section{Sample Characteristics}

Sample characteristics were similar at each wave of data collection. In 2014, $40.7 \%$ of children surveyed were non-Hispanic white and $44.6 \%$ were Hispanic. Sixty-three percent of those surveyed spoke English as the primary language at home, 29.7\% spoke Spanish, and 4.8\% spoke a primary language other than Spanish or English. Almost one-third of participants reported a household income of $<\$ 24,999$ per year, $24.2 \%$ reported an income of $\$ 25,000$ $\$ 48,999$ per year, $14.2 \%$ reported an income of $\$ 49,000-\$ 81,999$ per year, and $25.3 \%$ reported an income $>\$ 82,000$ per year. Approximately $24.8 \%$ of children in the study attended a California State preschool, $49.1 \%$ attended a private preschool, $4.3 \%$ attended a home preschool, and $20.0 \%$ attended a Head Start preschool.

Overweight and Obesity Prevalence and Trends between 2006 and 2014

Figure 1 displays the prevalence of overweight and obesity at the 2006, 2009/10, and 2014 assessments. When compared to the baseline sample (collected in 2006), the odds of OW/OB were 1.50 (95\% CI: $1.15,1.96)$ times higher for children assessed in 2009/10 and 1.42 (95\% CI: $1.15,1.96)$ times higher for children assessed in 2014. Odds of OW/OB for children assessed in 2014 were not significantly different from children assessed in 2009/10 (OR $=0.95 ; 95 \%$ CI: $0.73,1.23)$.

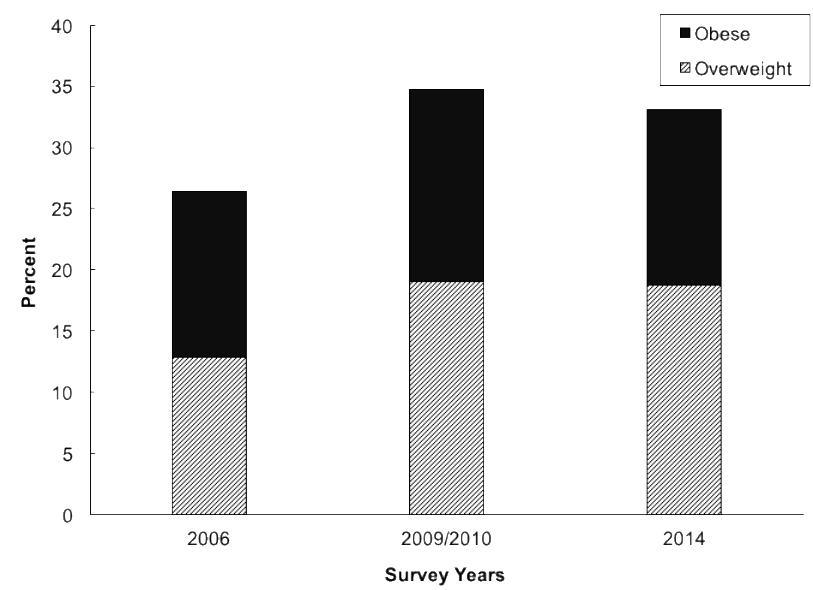

Figure 1. Prevalence of Overweight and Obesity for Preschool-Age Children in San Luis Obispo County, 2006-2014

\section{Sociodemographic Correlates of Overweight and Obesity for Children Assessed in 2014}


In 2014, odds of OW/OB did not differ between boys and girls $(\mathrm{p}=.43)$. In bivariate models, a number of sociodemographic correlates of OW/OB were noted in Table 1. Odds of OW/OB were 2.21 times higher for Hispanic children than non-Hispanic white children $(\mathrm{p}<.001)$; no difference in odds of $\mathrm{OW} / \mathrm{OB}$ was observed between non-Hispanic white and children from other races/ethnicities. Odds of OW/OB for children who attended preschool in the southern part of SLO County were 1.68 times higher than children who attended preschool in northern SLO County $(p=.002)$; odds of OW/OB for children attending preschools in other regions of the county were not statistically different from children attending preschool in northern SLO
County. With regard to preschool program, odds of OW/OB for children who attended a Head Start preschool program were 2.87 times higher than children who attended private preschools ( $\mathrm{p}$ $=.002$ ). Odds of OW/OB for children from families with incomes less than $\$ 25,000$ or between $\$ 25,000-\$ 48,999$ were 1.69 times and 2.01 times higher than children with family incomes of more than $\$ 82,000$, respectively.

Table 1 presents findings from the adjusted, multivariable model. In this model, odds of $\mathrm{OW} / \mathrm{OB}$ were significantly higher for children attending preschool in SLO City and who participated in a California State or Head Start preschool.

Table 1

Prevalence and Predictors of Overweight and Obesity in 2014

\begin{tabular}{|c|c|c|c|c|c|}
\hline & $\begin{array}{c}\text { Total } \\
\text { Sample }\end{array}$ & $\begin{array}{l}\text { Healthy } \\
\text { Weight }\end{array}$ & $\begin{array}{l}\text { Overweight/ } \\
\text { Obese }\end{array}$ & $\begin{array}{c}\text { Bivariate Prediction of } \\
\text { OW/OB }\end{array}$ & $\begin{array}{c}\text { Multivariate Prediction of } \\
\mathrm{OW} / \mathrm{OB}\end{array}$ \\
\hline Race/Ethnicity & $\mathrm{n}(\%)$ & $\mathrm{n}(\%)$ & $\mathrm{n}(\%)$ & OR (CI) & $\mathrm{AOR}(\mathrm{CI})$ \\
\hline Non-Hispanic/White & $40.7(180)$ & $30.3(134)$ & $10.4(46)$ & Ref & Ref \\
\hline Hispanic White & $44.6(197)$ & $25.4(112)$ & $19.2(85)$ & $2.21(1.43,3.44)$ & $1.54(0.86,2.79)$ \\
\hline Other & $12.9(57)$ & $9.5(42)$ & $3.4(15)$ & $1.04(0.52,2.02)$ & $0.88(0.41,1.82)$ \\
\hline Not reported & $1.8(8)$ & & & & \\
\hline \multicolumn{6}{|c|}{ Region of San Luis Obispo County } \\
\hline North County & 33.7 (149) & $24.4(108)$ & $9.3(41)$ & Ref & Ref \\
\hline South County & $31.9(141)$ & $19.5(86)$ & $12.4(55)$ & $1.68(1.03,2.77)$ & $1.72(0.97,3.07)$ \\
\hline San Luis Obispo City & $24.4(108)$ & $16.3(72)$ & $8.1(36)$ & $1.32(0.77,2.27)$ & $2.90(1.35,6.40)$ \\
\hline Los Osos/Morro Bay & $8.2(36)$ & $5.0(22)$ & $3.2(14)$ & $1.68(0.77,3.56)$ & $1.68(0.66,4.19)$ \\
\hline Not reported & $1.8(8)$ & & & & \\
\hline \multicolumn{6}{|l|}{ Type of School } \\
\hline Private & $49.1(217)$ & $36.4(161)$ & $12.7(56)$ & Ref & Ref \\
\hline California State & $24.8(110)$ & $15.8(70)$ & $9.0(40)$ & $1.64(1.00,2.69)$ & $2.36(1.06,5.32)$ \\
\hline Head Start & $20.0(88)$ & $10.0(44)$ & $10.0(44)$ & $2.87(1.72,4.84)$ & $2.76(1.20,6.45)$ \\
\hline Home & $4.3(19)$ & $2.9(13)$ & $1.4(6)$ & - & - \\
\hline Not reported & $1.8(8)$ & & & & \\
\hline \multicolumn{6}{|l|}{ Annual family income } \\
\hline$>\$ 82,000$ & $25.3(112)$ & $19.2(85)$ & $6.1(27)$ & Ref & Ref \\
\hline$\$ 49,000-\$ 81,999$ & $14.2(63)$ & $10.6(47)$ & $3.6(16)$ & $0.94(0.46,1.85)$ & $0.78(0.36,1.65)$ \\
\hline$\$ 25,000-\$ 48,999$ & $24.2(107)$ & $14.0(62)$ & $10.2(45)$ & $2.01(1.17,3.48)$ & $1.11(0.53,2.29)$ \\
\hline$<\$ 25,000$ & $29.9(132)$ & $18.6(82)$ & $11.3(50)$ & $1.69(1.01,2.87)$ & $0.94(0.46,1.90)$ \\
\hline Not reported & $6.3(28)$ & & & & \\
\hline
\end{tabular}

${ }^{a}$ Analyses limited to children assessed $2014(n=442)$ 


\section{Discussion}

Our findings illustrate prevalence of and trends for $\mathrm{OW} / \mathrm{OB}$ among preschool-age children in SLO County are similar to state and national data (Skinner et al., 2018). In the present study, we noted that in $2006, \sim 27 \%$ of preschool-age children in SLO County were classified as OW/OB, increasing to $\sim 35 \%$ in $2009 / 10$. The prevalence of OW/OB in 2014 was $~ 33 \%$, which was not significantly different from prevalence of OW/OB in 2009/10. These trends mirror state-level trends, with most recent surveys illustrating $\sim 30 \%$ of California children are overweight or obese (Trust for American's Health \& Robert Wood Johnson Foundation, 2018), but also illustrating leveling-off, or even declines, in the prevalence of overweight and obesity among preschool-age children (Trust for American's Health \& Robert Wood Johnson Foundation, 2018). National-level data show similar increases in prevalence of $\mathrm{OW} / \mathrm{OB}$ among children aged 2-5 years in the early 2000 's, followed by decreases between 2009/10 and 2013/14 surveys (Ogden et al., 2016). Although promising, it is important to note that the prevalence of OW/OB both locally and nationally are still well-above public health targets (Office of Disease Prevention and Health Promotion, 2015), suggesting continued surveillance and prevention efforts are needed.

Despite this promise, our findings also indicate certain populations likely need targeted prevention and intervention efforts to decrease prevalence of and risk for OW/OB. Consistent with previous findings (Babey, Hastert, Wolstein, \& Diamant, 2010; Pan, Blanck, Sherry, Dalenius, \& Grummer-Strawn, 2012), unadjusted, bivariate analyses suggested significant sociodemographic disparities existed for prevalence of $\mathrm{OW} / \mathrm{OB}$. These analyses revealed children who were Hispanic, residing in low income households, or attending a preschool in the southern part of SLO County or that was part of the Head Start preschool program were at higher risk for OW/OB. However, in the fullyadjusted multivariable model, only region (attending a preschool in SLO City) and preschool type (California State or Head Start) were associated with increased risk for OW/OB.
This heightened risk for OW/OB by region and preschool type reflect state- and national-level data, which illustrate $\mathrm{OW} / \mathrm{OB}$ disproportionately affects communities with lower sociodemographic status (Ogden et al., 2018; Ogden, Lamb, Carroll, \& Flegal, 2010; Pan, May, Wethington, Dalenius, \& GrummerStrawn, 2013; Trust for American's Health \& Robert Wood Johnson Foundation, 2018). In particular, rates of poverty in SLO City are $32.7 \%$, with a median household income of $\$ 47,777$, which is lower than poverty rates $(11.0 \%)$ and median household incomes $(\$ 64,014)$ across SLO County (U.S. Census Bureau, 2017). Additionally, California State and Head Start preschools both serve lowincome families (Administration for Children \& Families \& Services, 2018; San Luis Obispo County Office of Education, 2018), which are more often comprised of racial/ethnic minorities and headed by parents with low educational attainment (Williams, Priest, \& Anderson, 2016). That sociodemographic characteristics, such as income and ethnicity, were not significant predictors in the adjusted, multivariable model is intriguing given consistent associations between these factors and obesity risk (Babey et al., 2010; Pan et al., 2012); however, it is possible that region and enrollment in a State or Head Start preschool are more robust indicators of lower sociodemographic status given well-documented bias in self-reported sociodemographic data (Moore, Stinson, \& Welniak, 1997).

The association between sociodemographic status and obesity risk seen in the present study are likely mediated and moderated by a number of risk and protective factors, such as access to healthy food, opportunities for physical activity, and health care. Identification of county regions and preschools wherein prevalence of childhood OW/OB is highest provides an important foundation for targeted prevention and intervention efforts. Further research within these settings is needed to understand the most effective modifiable targets for programs aimed at reducing $\mathrm{OW} / \mathrm{OB}$ prevalence among preschoolers in SLO County. 


\section{Limitations}

The findings of this study should be interpreted within the context of its limitations. This study focused on a small, rural county in California. A benefit of this focus was that the county is characterized by significant financial and social disparity; however, this focus may limit the generalizability of our findings. Generalizability is further limited because the sample was a convenience sample of young children attending preschool. Additionally, parents self-reported their children's demographic data; as with any type of self-reported data, it is possible that these data were biased due to misreporting.

\section{Conclusions}

Our findings underline the importance of continued surveillance of OW/OB trends among preschool-age children in SLO County. Although our examination of changes in obesity prevalence between 2006 and 2014 suggest that rates of $\mathrm{OW} / \mathrm{OB}$ are leveling, prevalence is still higher than national targets, which suggests further prevention and intervention efforts are needed. Further research within SLO City and California State and Head Start preschools is needed to better understand mediators and moderators of associations between preschool region and type and children's risk for OW/OB. Multilevel interventions to reduce childhood obesity should target the modifiable factors that influence young children's risk for obesity and encourage healthier lifestyle choices for children and families. It is important for these interventions to facilitate environments that supports optimal education, healthy dietary intake, and promotion of physical activity in young children, their families, and their preschool settings.

\section{References}

Administration for Children \& Families, \& Services, U.S. Department of Health and Human Services. (2018). Office of Head Start: An Office of the Administration for Children \& Families. Retrieved from https://www.acf.hhs.gov/ohs

Babey, S.H., Hastert, T.A., Wolstein, J., \& Diamant, A.L. (2010). Income disparities in obesity trends among California adolescents. American Journal of Public Health, 100(11), 2149-2155. doi:10.2105/AJPH.2010.192641

Centers for Disease Control and Prevention. (2007). National Health and Nutrition Examination Survey (NHANES): Anthropometry Procedures Manual. Retrieved from: https://www.cdc.gov/nchs/data/nhanes/nhanes_07_08/manual_an.pdf

Freedman, D.S., Khan, L.K., Serdula, M.K., Dietz, W.H., Srinivasan, S.R., \& Berenson, G.S. (2005). The relation of childhood BMI to adult adiposity: The Bogalusa Heart Study. Pediatrics, 115(1), 2227. doi:10.1542/peds.2004-0220

Jankovitz, K.Z., McGaughey, K.J., Torn, K., Ravalin, D., \& McDermott, A.Y. (2012). Prevalence of overweight and obesity in Hispanic Preschool Children in San Luis Obispo County, California. Californian Journal of Health Promotion, 10, 114-121.

Jones-Smith, J.C., Dieckmann, M.G., Gottlieb, L., Chow, J., \& Fernald, L.C. (2014). Socioeconomic status and trajectory of overweight from birth to mid-childhood: The Early Childhood Longitudinal Study-Birth Cohort. PLoS ONE, 9(6), e100181. doi:10.1371/journal.pone.0100181

Moore, J.C., Stinson, L.L., \& Welniak, E.J. (1997). Income measurement error in surveys: A review. Retrieved from Washington, D.C.: https://www.census.gov/srd/papers/pdf/sm97-05.pdf

National Center for Health Statistics. (2016). Health, United States, 2015: With Special Feature on Racial and Ethnic Disparities. Retrieved from: https://www.cdc.gov/nchs/data/hus/hus15.pdf

Office of Disease Prevention and Health Promotion. (2015). Healthy People 2020. Retrieved from: https://www.healthypeople.gov/

Ogden, C.L., Carroll, M.D., Fakhouri, T.H., Hales, C.M., Fryar, C.D., Li, X., \& Freedman, D.S. (2018). Prevalence of Obesity Among Youths by Household Income and Education Level of Head of 
Household - United States 2011-2014. MMWR Morb Mortal Wkly Rep, 67(6), 186-189. doi:10.15585/mmwr.mm6706a3

Ogden, C.L., Carroll, M.D., Lawman, H.G., Fryar, C.D., Kruszon-Moran, D., Kit, B.K., \& Flegal, K.M. (2016). Trends in obesity prevalence among children and adolescents in the United States, 19881994 Through 2013-2014. Journal of the American Medical Association, 315(21), 2292-2299. doi:10.1001/jama.2016.6361

Ogden, C.L., Lamb, M.M., Carroll, M.D., \& Flegal, K.M. (2010). Obesity and socioeconomic status in children and adolescents: United States, 2005-2008. NCHS Data Brief(51), 1-8.

Pan, L., Blanck, H.M., Sherry, B., Dalenius, K., \& Grummer-Strawn, L.M. (2012). Trends in the prevalence of extreme obesity among US preschool-aged children living in low-income families, 1998-2010. Journal of the American Medical Association, 308(24), 2563-2565. doi:10.1001/jama.2012.108099

Pan, L., May, A.L., Wethington, H., Dalenius, K., \& Grummer-Strawn, L.M. (2013). Incidence of obesity among young U.S. children living in low-income families, 2008-2011. Pediatrics, 132(6), 10061013. doi:10.1542/peds.2013-2145

San Luis Obispo County Office of Education. (2018). California State Preschools/First 5 Preschools. Retrieved from: https://www.slocoe.org/divisions/educational-support-services/early-learningand-educational-support/california-state-preschools-first-5-preschools/

Skinner, A.C., Ravanbakht, S.N., Skelton, J.A., Perrin, E.M., \& Armstrong, S.C. (2018). Prevalence of obesity and severe obesity in US children, 1999-2016. Pediatrics. doi:10.1542/peds.2017-3459

Trust for American's Health, \& Robert Wood Johnson Foundation. (2018). The State of Obesity in California. Retrieved from: https://stateofobesity.org/states/ca/

U.S. Census Bureau. (2017). State \& County QuickFacts: San Luis Obispo, CA. Retrieved from: https://www.census.gov/quickfacts/fact/table/US/PST045217

Wang, Y., \& Beydoun, M.A. (2007). The obesity epidemic in the United States--gender, age, socioeconomic, racial/ethnic, and geographic characteristics: a systematic review and metaregression analysis. Epidemiologic Reviews, 29, 6-28. doi:10.1093/epirev/mxm007

Williams, D.R., Priest, N., \& Anderson, N.B. (2016). Understanding associations among race, socioeconomic status, and health: Patterns and prospects. Health Psychology, 35(4), 407-411. doi:10.1037/hea0000242

Author Information

Alison K. Ventura, Ph.D., CLEC

Department of Kinesiology and Public Health

California Polytechnic State University, San Luis Obispo

One Grand Ave

San Luis Obispo, CA 93407

(805) 756-5693

akventur@calpoly.edu 